\title{
Entre mémoire nationale et mémoire familiale. Les films de la vague sépia sous la France de Nicolas Sarkozy
}

Face aux rapports complexes entre politique, mémoires et médias', il est fréquent de focaliser l'attention sur un secteur particulier tantôt en privilégiant l'analyse de la politique mémorielle telle qu'elle se construit au niveau de l'État,

\author{
L'auteur : Sébastien FeVRY \\ est Chargé de cours à \\ l'École de Communication \\ (COMU, Université catho- \\ lique de Louvain).
} tantôt en travaillant sur des productions culturelles comme le cinéma ou la photographie pour y interroger le type de mémoire véhiculée 2 . Si ces approches sont éminemment valables et productives - il ne s'agit nullement de les remettre en cause ou de contester leur pertinence -, il me paraît toutefois qu'elles échouent souvent à prendre en compte les interactions entre les différents aspects de la vie sociale et à montrer comment les enjeux mémoriels découlent toujours de conjonctures singulières nouant aussi bien des paramètres politiques et médiatiques ${ }^{3}$ que familiaux et culturels.

C'est pourquoi je voudrais privilégier ici une approche inspirée du concept d'articulation développé par Stuart Hall et les Cultural Studies. Pour Hall, ce concept constitue « un moyen de comprendre comment des éléments idéologiques en viennent, sous certaines conditions, à former un ensemble cohérent dans un discours, ainsi qu'un moyen de s'interroger sur la façon dont ils sont ou non articulés à des conjonctures spécifiques et à certains sujets politiques ${ }^{4}$. Pour

'Cet article est issu d'une communication intitulée "Le cinéma couleur sépia sous la France de Nicolas Sarkozy. Nostalgie et identité nationale » faite dans le cadre de la journée d'étude « Media, Memory and Nostalgia » organisée par le French Media Research Group, Newcastle, octobre 2013.

${ }^{2}$ Pour la première approche, voir notamment GensBURGER Sarah, Les Justes de France. Politiques publiques de la mémoire, Paris, Presses de Sciences Po, 2010, 239 p. Pour la seconde approche, le lecteur pourra se rapporter à LANDSBERg Alison, Prosthetic Memory: The Transformation of American Remembrance in the Age of Mass Culture, New York, Columbia University Press, 2004, 240 p.

3 Parmi les approches articulant les sphères politique et cinématographique dans la construction d'une mémoire collective au sein de la société française, on peut citer l'essai précurseur de Henry Rousso Le syndrome de Vichy (notamment le chapitre intitulé Le miroir brisé) ainsi que l'ouvrage de Sylvie Lindeperg Les écrans de l'ombre. Rousso Henry, Le syndrome de Vichy. De 1944 à nos jours, Paris, Seuil, 1987, 414 p. LINDEPERG Sylvie, Les écrans de l'ombre. La Seconde Guerre mondiale dans le cinéma français (1944-1969), Paris, CRNS Éditions, 2001, 443 p

${ }^{4}$ Hall Stuart, cité par Cervulle Maxime, "La politique des différences ", in Hall Stuart, Identités et cultures 2. Politiques des différences, in Cervulle Maxime (dir.), Paris, Éditions Amsterdam, 2013, p. 14. 
qui s'intéresse à l'analyse des médias, cette approche est extrêmement profitable dans la mesure où elle confie un rôle central aux productions médiatiques et culturelles, puisque celles-ci sont comprises comme un lieu d'articulation "entre des formations discursives et des formations sociales $»^{5}$, comme un point où viennent à la fois se sédimenter des discours et s'organiser des pratiques.

Le concept d'articulation est particulièrement précieux pour saisir les enjeux des films de la vague sépia qui ont culminé en France lors de la présidence de Nicolas Sarkozy et dont la composante nostalgique est étroitement chevillée à la valorisation d'un passé idéalisé. Mon propos ne sera pas seulement d'établir les caractéristiques formelles de la vague sépia, mais bien de montrer comment le type de mémoire qu'elle met en place s'articule avec la politique mémorielle promue par le gouvernement Sarkozy, une politique qui tend elle aussi à se focaliser sur des questions d'identité et de valorisation du passé national. Bref, il s'agira de situer le cinéma sépia dans une relation mémorielle dynamique qui combine à la fois la production, la configuration et l'insertion des films dans la société française du milieu des années 2000.

Ce faisant, je voudrais également souligner le rôle du cinéma comme lieu d'articulation majeur dans le contexte d'une société médiatique qui multiplie les pratiques et les supports mémoriels ${ }^{6}$. À mon sens, la vague du cinéma sépia atteste nettement de la centralité de la forme cinématographique dans les enjeux de mémoire, même si d'autres productions médiatiques comme des émissions télévisées ou des séries ont pu étoffer le succès de la mode sépia auprès du public ${ }^{7}$. Par ailleurs, et ce sera là l'un des objectifs principaux de cet article, on s'attachera aussi à montrer comment les films de la vague sépia témoignent d'un basculement assez net dans la sélection et l'activation des cadres mémoriels entourant la remémoration du passé national.

\section{Le cinéma sépia sous l’angile de sa réception médiatique}

Au cours des années 2000 , un nombre important de films empreints d'une dimension nostalgique surgissent sur les écrans français : Les Triplettes de Belleville (Chomet, 2003), Le Petit Nicolas

\footnotetext{
${ }^{5}$ Cervvulle Maxime, «La politique des différences », op. cit., p. 16.

${ }^{6}$ À ce sujet, voir notamment Hoskins Andrew, "Media, Memory, Metaphor: Remembering and the Connective Turn ", Parallax, 2011, vol. 17, n 4, pp. 19-31, disponible à l'adresse suivante : http://www.tandfonline.com/ (consultée le 14 août 2014).

${ }_{7}$ On peut par exemple penser à l'émission de téléréalité Le Pensionnat de Chavagnes, diffusée à partir de septembre 2004 sur M6 (soit dans la foulée de la sortie des Choristes au cinéma) et qui consistait à plonger des collégiens d'aujourd'hui dans le cadre d'un pensionnat des années 50.
} 
(Tirard, 2009), Un long dimanche de fiançailles (Jeunet, 2004), La Nouvelle guerre des boutons (Barratier, 2011)... Très vite, surtout après le succès surprise des Choristes de Christophe Barratier en 2004, de nombreux critiques qualifient ces films de cinéma aux couleurs sépia, faisant ainsi référence à l'aspect qu'ont les anciennes photographies lorsque leurs tirages tirent trop fort sur le brun. Dans cette perspective, l'adjectif "sépia " renvoie au fait que le goût passéiste de ces productions se retrouve notamment dans la composition des images, dans leurs teintes un peu passées.

Par rapport à cette dénomination médiatique, le premier point à souligner est que l'intitulé " cinéma sépia » ne constitue nullement la partie émergente d'un discours articulé que l'on retrouverait dans la presse généraliste ou dans certains magazines spécialisés comme Les Cahiers du cinéma ou Positif. On est loin ici du travail critique effectué par Michel Foucault ou Serge Daney dans les années 1970 autour de la vague du cinéma rétro, emblématisé par des films comme Lacombe Lucien (1974) de Louis Malle ou Portier de nuit (1974) de Liliana Cavani.

Dans la plupart des cas, le terme "sépia " est simplement utilisé pour appuyer la critique de films populaires jugés comme passéistes. Par exemple, dans L'Express du 3 mai 2004, Les Choristes est qualifié de "fable sépia à rebrousse-poil de la modernité ". Au sujet du même titre, Le Parisien note que le film éveille "les arômes sépia, subtilement envoûtants de la nostalgie $»^{10}$. Sur Internet, un blogueur intitule sa chronique du film de Laurent Tirard Le Petit Nicolas : "Une France couleur sépia "". Et lorsque Virginie Guichard, dans sa critique du Guardian, parle des Choristes comme d'un film "sepia-tinted "12, c'est pour le rapprocher de productions comme Amélie Poulain (Jeunet, 2001), Les Triplettes de Belleville de Sylvain Chomet ou Un long dimanche de fiançailles de Jean-Pierre Jeunet. Pour son film Micmacs à tire-larigot (2009), le même Jean-Pierre Jeunet sera accusé

${ }^{8}$ La mouvance du cinéma rétro a été critiquée par Michel Foucault dans un entretien donné aux Cahiers du cinéma en juillet-août 1974. Contrairement aux films de la vague sépia, les films de la mode rétro ne visent pas à reconstituer le souvenir d'un passé heureux, mais plutôt, comme l'a montré Foucault, à évacuer le souvenir des luttes populaires en présentant l'image d'une France démystifiée où il n'y a ni véritable héros ni véritable salaud. L'essentiel de cet entretien est repris dans l'ouvrage que Patrice Maniglier et Dork Zabunyan ont consacré aux rapports entre Foucault et le cinéma. FoucaUlt Michel, "Anti-Rétro », in MANIGLIER Patrice, ZABUnYAN Dork, Foucault va au cinéma, Paris, Bayard, 2011, pp. 129-135.

${ }^{9}$ "Tous un peu Jugnot", page publiée sur le site de L'Express, mai 2004, disponible à l'adresse suivante: http://www.lexpress.fr/ (consultée le 5 mai 2014).

10 VAVAsseur Pierre, "Les Choristes enchante ", page publiée sur le site du Parisien, mars 2004, disponible à l'adresse suivante : http://www.leparisien.fr/ (consultée le 5 mai 2014).

"ARTUSO Vincent, "Une France couleur sépia », page publiée sur le site de Woxx, octobre 2010, disponible à l'adresse suivante: http://www.woxx.lu/id_article/3144 (consultée le 5 mai 2014).

${ }^{12}$ Guichard Virginie, "Songs of Praise », page publiée sur le site du Guardian, mai 2005, disponible à l'adresse suivante: http://www.theguardian.com/ (consultée le 5 mai 2014). 
par un journaliste de France Inter de pédaler « dans une semoule sépia vaguement nostalgique des années $50 \Perp^{13}$.

Bref, dans son acception la plus large, l'adjectif " sépia » semble pouvoir s'associer à tout film français des années 2000 reposant sur une conception assez nostalgique du passé et évoquant auprès du spectateur la mémoire d'une France disparue.

\section{Une première définition du cinéma sépia}

Si cette première approche du cinéma sépia permet de mesurer l'implantation du terme dans la presse et de vérifier ainsi l'existence d'une conception partagée du phénomène, elle ne nous renseigne que modérément sur la configuration des films et le type de mémoire qu'ils sont capables de susciter auprès du public. Pour aller plus loin dans la définition de la vague sépia, on peut dégager deux grandes caractéristiques permettant de mettre en avant les œuvres les plus significatives du genre.

Tout d'abord, il paraît évident que les productions emblématiques du cinéma sépia conjuguent la France au passé. Pouvant encore être transmis par les souvenirs familiaux, ce passé est relativement proche et relève d'une mémoire intergénérationnelle s'étendant sur trois ou quatre générations. C'est pourquoi le cinéma sépia, dans son viseur historique, a comme borne ultime la guerre 1914-1918 telle qu'elle est mise en scène, par exemple, dans Un long dimanche de fiançailles de Jean-Pierre Jeunet. Le cinéma sépia porte aussi sur les années de la Seconde Guerre mondiale (chez Barratier dans La Nouvelle guerre des boutons) mais, plus fréquemment encore, sur la période des Trente Glorieuses (1945-1973), période que l'on voit apparaître dans Les Triplettes de Belleville, Le Petit Nicolas, la deuxième version de La Guerre des boutons (Samuell, 2011) dont l'intrigue se déroule au début des années 1960 pendant la guerre d'Algérie, ou dans Populaire (2012), un film de Régis Roinsard mettant en scène des concours de vitesse dactylographique dans la France des années 1950.

Le cinéma sépia se caractérise ensuite par une forte dimension intertextuelle. II ne se contente pas de mettre en scène un passé proche, il réactualise aussi des modes de représentation issus du passé. Parfois, il s'agit d'un remake pur et simple. Les Choristes est adapté du film de Jean Dréville La Cage au rossignol (1945) qui avait remporté un large succès public au lendemain de la Deuxième Guerre

${ }^{13}$ DeLMAS Laurent, «Irene encore et encore », blog publié sur le site de France Inter, octobre 2009, disponible à l'adresse suivante : http://www.franceinter.fr/ (consultée le 5 mai 2014). 
mondiale. Le Petit Nicolas adapte à l'écran la série de récits pour la jeunesse signés par René Goscinny et illustrés par Jean-Jacques Sempé dont les premières publications remontent à la fin des années 1950. Les deux nouvelles versions de La Guerre des boutons (2001) transposent le célèbre roman de Louis Pergaud (1912) qui avait déjà été porté à l'écran par Yves Robert en 1962. Parfois, l'aspect intertextuel est moins prégnant et se manifeste sous la forme d'emprunts ponctuels ou d'hommages. Avec Les Triplettes de Belleville, Sylvain Chomet construit un univers qui doit beaucoup à Jacques Tati, référence que le réalisateur rendra encore plus manifeste avec L'lllusionniste (2010). Pareillement, lorsqu'il tourne Faubourg 36 (2008), Christophe Barratier montre son admiration pour le cinéma du Front populaire et les œuvres de Marcel Carné et de Julien Duvivier.

\section{Mémoire et nostalgie}

C'est en partant de ces deux caractéristiques que l'on peut commencer à envisager le travail de mémoire et le sentiment de nostalgie déployés par les films auprès des spectateurs français des années 2000. Cependant, pour avancer davantage dans cette direction, il convient de mieux préciser les rapports qu'entretiennent mémoire et nostalgie. Si les deux sont intimement liées, elles ne se recouvrent pas entièrement. Dans son acception littérale, la nostalgie a un sens plus spatial que temporel, puisque étymologiquement le terme renvoie au désir du retour à la maison. Autrefois, ce terme servait à désigner l'étrange affection qui frappait les soldats en contrée étrangère et qui se caractérisait par une profonde dépression liée à l'éloignement de la terre natale ${ }^{14}$. Dans ce processus, la mémoire jouait un rôle déterminant, puisque le désir de retour vers une patrie perdue ne pouvait être déclenché que par le processus mémoriel lui-même et certaines de ses manifestations comme des souvenirs ou des hallucinations.

En vue d'une compréhension plus fine des rapports entre mémoire et nostalgie, il est utile de partir de la distinction proposée par Svetlana Boym dans son ouvrage The Future of Nostalgia. L'auteure distingue deux grands types de nostalgie : la nostalgie restauratrice et la nostalgie réflexive. La première est centrée sur le foyer et vise à restaurer la communauté perdue. II s'agit d'une nostalgie qui peut être utilisée à des fins nationalistes et qui repose sur la réaffirmation de la tradition, le retour au terroir et la quête d'un passé authentique. Pour la nostal-

${ }_{14}$ Pour un histoire culturelle de la nostalgie, le lecteur se rapportera aux deux premiers chapitres de l'ouvrage The Future of Nostalgia. Boym Svetlana, The Future of Nostalgia, New York, Basic Books, 2001, pp. 3-32. 
gie restauratrice, «le passé n'est supposé présenter aucun signe de déclin ou de décadence; il doit être fraîchement peint dans son 'image d'origine' et rester éternellement jeune ${ }^{15}$. La nostalgie réflexive est, quant à elle, une nostalgie qui joue plutôt sur la rêverie et qui ne prétend nullement reconstruire le passé à l'identique. Cette forme de nostalgie tire sa force créative du fossé infranchissable séparant le passé du présent et fonctionne comme "une méditation sur l'histoire et le passage du temps ${ }^{16}$.

Ces tendances nostalgiques peuvent s'associer à deux types de mémoire qui sont respectivement la mémoire container (containered memory) et la mémoire transculturelle (transcultural memory), appelée aussi travelling memory. Ce double concept est très opérationnel dans le champ actuel des Memory Studies, chez Astrid Erll notamment ${ }^{17}$. Pour Erll, la mémoire container désigne une mémoire qui épouse souvent les contours de l'État-nation, mais qui renvoie plus largement à une mémoire fermée sur elle-même, homogène et rétive à accueillir tout élément extérieur. Il y a ici comme un phénomène d'isomorphisme et de renforcement mutuel entre la mémoire, le territoire et la communauté. Plutôt que container, on pourrait appeler cette mémoire "centripète ". La mémoire transculturelle désigne quant à elle une mémoire en mouvement, c'est-à-dire une mémoire caractérisée par un trafic constant entre différentes cultures et différentes époques, une mémoire solidaire aussi des phénomènes de migration et de diaspora qui débordent les frontières des États-nations. Cette mémoire transculturelle, on pourrait l'appeler «mémoire centrifuge».

Comme on le pressent, il s'avère tout à fait productif d'associer la nostalgie restauratrice à une mémoire container et la nostalgie réflexive à une mémoire transculturelle. Et de fait, la première forme de nostalgie tendra beaucoup plus à se fédérer autour d'une mémoire centripète, alors que la deuxième forme jouera plutôt sur le décalage et l'éparpillement des souvenirs. Autrement dit, la rêverie de la nostalgie réflexive tirera profit des allers-retours d'une mémoire voyageuse et s'accommodera difficilement de l'image statique du passé promue par une mémoire renfermée sur elle-même.

\footnotetext{
${ }^{15}$ Boym Svetlana, The Future of Nostalgia, op. cit., p. 49 [traduction de l'auteur].

$16 \mathrm{lbid}$. [traduction de l'auteur].

${ }^{17}$ ERLL Astrid, «Travelling Memory », Parallax, 2011, vol. 17, n 4, pp. 4-18, disponible à l'adresse suivante: http:// www.tandfonline.com/ (consultée le 5 mai 2014).
} 


\section{La nostalgie restauratrice du cinéma sépia}

Dans le cas de la vague sépia, et si l'on excepte certaines productions, comme Les Triplettes de Belleville, qui relèvent davantage de la nostalgie réflexive, force est de constater que la plupart des films se rangent plutôt du côté d'une nostalgie restauratrice, elle-même soutenue par une mémoire container ou centripète.

La promotion d'une telle mémoire passe tout d'abord par une simplification du passé qui contraste avec la complexité du temps présent où se tient le spectateur. Les films sont débarrassés de tout élément qui pourrait évoquer les difficultés de l'époque contemporaine. La France des Choristes, de La Nouvelle guerre des boutons ou du Petit Nicolas est une France blanche, sans soulèvement de banlieues, où n'apparaît nulle figure de l'étranger qui viendrait rappeler aux spectateurs certains débats autour de l'immigration. Le réalisateur des Choristes, Christophe Barratier, le reconnaît d'ailleurs candidement. Dans une interview, il expliquait qu'il n'avait jamais songé à transposer Les Choristes à l'époque actuelle, car il lui aurait fallu intégrer des problèmes " comme les cités HLM, le chômage chronique, l'assimilation ou la délinquance juvénile ${ }^{18}$.

Mais les films de la vague sépia ne se contentent pas d'évacuer de leur représentation du passé tout élément perturbateur pour le temps présent. Plus fondamentalement, ces films sont profondément a-historiques, c'est-à-dire qu'ils confrontent très peu leurs personnages à ce que l'on pourrait appeler le sens de l'histoire. Les protagonistes se tiennent toujours à distance des grands événements qui ont bouleversé le siècle et leurs aventures ne sérigent en rien comme le paradigme d'un destin collectif qui révélerait le devenir d'un peuple ou d'une communauté'19.

À cet égard, les films de Christophe Barratier sont intéressants à observer. Seul La Nouvelle guerre des boutons se déroule pendant la Deuxième Guerre mondiale et au premier abord, il est vrai que l'on y célèbre la Résistance puisque les deux villages ennemis se réconcilient pour faire passer de l'autre côté de la frontière une jeune fille juive réfugiée dans la région. Cependant, cette opération, pour héroïque qu'elle soit, se passe en l'absence presque complète des nazis. Dans le film, l'armée allemande surgit seulement dans un plan de nuit, sous la forme d'un camion et de deux motocyclettes. Pour le reste, le film 
oppose surtout une poignée de miliciens, présentés de façon excessivement caricaturale, et l'ensemble des villageois, tous ou presque résistants. Dans ces conditions, la veine épique s'avère très faible et d'ailleurs, l'évacuation de l'adolescente est vécue par le jeune héros comme une rupture amoureuse et non comme la sensation victorieuse du devoir accompli.

Le processus d'évitement de l'histoire était déjà présent dans les deux autres films de Barratier. Par exemple, dans Faubourg 36, une ellipse assez extraordinaire est faite sur les années de guerre. A la fin du récit, le spectateur passe sans transition de l'été 1936, marqué par les premiers congés payés, à décembre 1945, année qui voit le personnage principal sortir de prison et découvrir Paris libéré. Enfin, dans Les Choristes, dont l'action se passe dans l'immédiate après-guerre, apparaît un orphelin qui sera plus tard adopté par le surveillant joué par Gérard Jugnot. Seul le prénom de l'écolier (Pépinot) pourrait faire penser que ses parents étaient des réfugiés républicains espagnols, mais le film stoppe là son évocation d'une histoire qui va bien au-delà du pensionnat où sont enfermés les enfants.

La mise à l'écart de l'histoire va souvent de pair avec un resserrement sur la sphère intime et familiale, sur des problèmes d'ordre domestique et privé. Dans cette perspective, il n'est pas étonnant que les genres cinématographiques adoptés par la vague sépia soient principalement ceux du mélodrame et de la comédie familiale, des genres qui précisément font du foyer ou de ses dérivés (l'école par exemple) le lieu principal des aventures des personnages. Dans son célèbre ouvrage sur la naissance du mélodrame, Peter Brooks a bien montré comment le mélodrame rabattait les grandes actions héroïques, porteuses de sens tragique et épique, dans un cadre intérieur et domestique $^{20}$. À partir du moment où est repoussée la confrontation avec I'histoire, le film se replie sur l'intime, ce qui permet du même coup de montrer des effets dramatiques qui n'excèdent pas la sphère privée et qui restent cantonnés à une mémoire familiale. C'est de nouveau vrai pour les films de Barratier ou pour Le Petit Nicolas de Laurent Tirard; c'est vrai aussi dans Un long dimanche de fiançailles où la quête de l'héroïne pendant la guerre de 1914-1918 est entièrement centrée sur la recherche de son fiancé.

${ }^{20}$ Pour Brooks, le mélodrame naît dans le contexte de la Révolution française et vient remplacer la tragédie dans une ère qui voit s'effondrer les grandes institutions du sacré qu'étaient le monarque et l'Église. En ce sens, le mélodrame est une réponse à la perte du sens tragique : il s'agit de reconduire sur un niveau intime et familier les valeurs de justice et de vérité qui agitaient autrefois de plus hautes sphères métaphysiques. BRooks Peter, L'imagination mélodramatique. Balzac, Henry James, le mélodrame et le mode de l'excès, Paris, Garnier, 2010, 261 p. 
Par ailleurs, si le comique est aussi fréquemment convoqué dans les films de la vague sépia, c'est qu'il permet de jouer sur le plaisir de la reconnaissance dont parlait déjà Freud ${ }^{21}$ en présentant aux spectateurs des objets familiers du passé. C'est peut-être à ce niveau que l'on peut encore pointer un élément essentiel dans la configuration des films, à savoir l'attention excessive portée aux objets du quotidien. C'est comme si l'évacuation du mouvement de l'histoire était compensée par la célébration d'objets appartenant spécifiquement à l'espace culturel français : vêtements, voitures, électroménager... Dans cette veine s'illustre particulièrement Le Petit Nicolas où les objets deviennent les déclencheurs de certains gags, ainsi que le montre la séquence où Nicolas s'enferme dans la Peugeot 404 de ses parents. À mon sens, ce goût du détail concret ne doit pas tellement être imputé à une volonté de rigueur historique; il correspond davantage à la trouvaille de l'antiquaire et vise à présenter au spectateur la réplique exacte d'un objet familier et désormais mythique du passé.

\section{Succès populaire et appropriation mémorielle}

Les films de la vague sépia reposent donc sur l'évocation d'une mémoire extrêmement unifiée et homogène, laquelle a manifestement rencontré l'adhésion d'un large public. Pour la plupart, les productions de la vague sépia ont remporté en France de véritables succès populaires. En 2004, le film Les Choristes connaît un triomphe inespéré au box-office et devient le film français le plus vu de l'année avec plus de huit millions de spectateurs (8.451.680 entrées). En 2009, Le Petit Nicolas de Laurent Tirard est lui aussi le premier film français au box-office avec plus de cinq millions de spectateurs (5.520.194 entrées). Pour le reste, des films comme Faubourg 36 ou La Guerre de boutons de Yann Samuell se situent tous au-dessus de la barre du million de spectateurs $^{22}$.

C'est à ce niveau que l'on peut établir un premier travail d'articulation entre les films et leur public. Jusqu'à présent, la question de la mémoire était abordée de façon relativement abstraite, à partir des productions elles-mêmes, alors que le travail de remémoration n'est effectif que lorsque le spectateur s'approprie le contenu mémoriel configuré par les films. Par ailleurs, il faut garder à l'esprit que la remé-

${ }^{21}$ Pour Freud, le plaisir comique est lié à une épargne d'énergie facilitée notamment par le processus de reconnaissance et la découverte soudaine d'éléments familiers. FREUD Sigmund, Le mot d'esprit et sa relation à l'inconscient, Paris, Gallimard, 1988 [1905], 442 p.

${ }^{22}$ Ces différents résultats peuvent être consultés sur le site Wikipédia qui propose pour chaque année une rubrique intitulée Box-office France, laquelle centralise sous une forme accessible des données chiffrées en provenance des sites cbo-boxoffice.com et Internet Movie Database. 
moration individuelle se produit un nombre potentiellement infini de fois, en fonction de chaque spectateur qui regarde le film, et surtout qu'elle s'accomplit à chaque fois sur la base des mêmes images du passé.

Dès lors, si nous reprenons à notre compte la notion de prosthetic memory développée par Alison Landsberg en vue de spécifier les nouvelles déclinaisons de la mémoire à l'heure des médias de masse, il apparaît clairement que les films de la vague sépia constituent des artefacts proposant une expérience mémorielle à la fois sur un mode singulier et collectif ${ }^{23}$. Pour Landsberg, les mémoires prosthétiques se développent sur un plan privé, mais elles naissent de la rencontre entre des représentations du passé diffusées à large échelle et la singularité d'une expérience individuelle ${ }^{24}$.

De plus, entre le film et son spectateur, entre le niveau collectif et individuel, doit encore se glisser la prise en compte des cadres mémoriels ${ }^{25}$ qui ont précisément pour but de favoriser la médiation entre les représentations médiatiques et le travail de mémoire qui s'accomplit chez chaque spectateur. Pour ce qui est de la vague sépia, le cadre familial constitue sans nul doute la forme de médiation la plus pertinente en termes d'appropriation mémorielle.

Dans son ouvrage The Generation of Postmemory, Marianne Hirsch a souligné le rôle déterminant des formes de médiation familiale dans le cadre de la réappropriation de traumatismes historiques par des personnes, souvent les descendants des victimes, qui ne possèdent parfois qu'une connaissance lointaine et déformée des événements vécus par leurs parents. Pour Hirsch, la famille fonctionne comme un cadre de référence qui favorise l'intégration personnelle des souvenirs en même temps que leur partage sur une large échelle, y compris pour des personnes n'ayant pas nécessairement éprouvé les événements

\footnotetext{
${ }^{23}$ Pour Landsberg, le cinéma ainsi que les autres médias de masse ont également ceci de particulier qu'ils peuvent communiquer des expériences mémorielles à des spectateurs qui sont a priori étrangers au passé véhiculé par les films : «the turn to mass culture - to movies, experiental museums, television shows, and so forth - has made what was once considered a group's private memory available to a much broader public. In this process, memories have ceased to belong exclusively to a particular group and instead have become part of a common public domain ». LANDSBERG Alison, Prosthetic Memory: The Transformation of American Remembrance in the Age of Mass Culture, op. cit., p. 11.

${ }^{24} \mathrm{Je}$ traduis et paraphrase ici Alison Landsberg. LANDSBERg Alison, Prosthetic Memory: The Transformation of American Remembrance in the Age of Mass Culture, op. cit., p. 19.

${ }^{25}$ La notion de 'cadre mémoriel' employée ici fait référence aux cadres sociaux de la mémoire tels qu'ils ont été définis par Maurice Halbwachs et évoqués par Paul Ricœur dans La mémoire, l'histoire, l'oubli. Sans entrer dans une longue définition de cette notion, rappelons, avec Ricœur, que l'on ne se souvient jamais seul et que les souvenirs «nous font voyager de groupe en groupe, de cadre en cadre, tant spatiaux que temporels». En outre, la notion de cadre mémoriel implique que le groupe à l'intérieur duquel on se replace pour se souvenir fournit également un cadre de référence qui donne forme et cohérence (narrative notamment) à la matière première des souvenirs personnels. RIC氏UR Paul, La mémoire, l'histoire, l'oubli, Paris, Seuil, 2000, p. 149.
} 
en question. En tant que réservoir d'images et d'histoires, la mémoire familiale constitue en effet une structure relativement commune facilitant l'entrecroisement des mémoires; en tant que substrat éminemment personnel, elle permet aussi une implication forte et affective dans le passé 26 .

Une telle réflexion est valable pour les productions sépia qui opèrent pourtant dans un autre contexte que les œuvres à caractère traumatique analysées par Marianne Hirsch. En jouant sur une sphère privée et non particulièrement guerrière ou héroïque, les films sépia se connectent directement à la mémoire familiale des spectateurs, et cela d'autant mieux qu'aucun élément traumatique ne vient freiner ce processus. Le cadre familial permet de s'approprier des souvenirs que nous n'avons pas nécessairement vécus, mais que nous raccrochons sans trop de difficulté à nos propres souvenirs parce qu'ils partagent un même cadre de référence. En ce sens, le fait que les films de la vague sépia se centrent sur des lieux comme des écoles ou des pensionnats, en plus de la famille, participe à ce mouvement général. De la même manière, si la thématique de l'enfance est aussi couramment évoquée, à travers presque tous les films, c'est parce que les jeux de l'enfance constituent également un cadre commun de souvenirs.

L'idée d'une appropriation mémorielle par le biais du cadre familial amène à penser que la génération de spectateurs ayant contribué au succès des films n'est certainement pas le jeune public des blockbusters hollywoodiens. Les films de la vague sépia sont contemporains d'une tendance sociologique qui voit un bouleversement des équilibres générationnels dans la fréquentation des cinémas. Ainsi, selon une enquête récente du CNC réalisée en 2012, le pourcentage des spectateurs seniors tendrait progressivement à dépasser celui du public âgé de moins de 25 ans qui était jusque-là considéré comme le public principal des salles obscures ${ }^{27}$.

Cette évolution sociologique appuie l'hypothèse que le public des films sépia serait en grande partie constitué de cette génération plus mature qui se rend de plus en plus massivement dans les salles obscures. Même s'il est présomptueux de vouloir dresser un portrait-ro-

${ }^{26}$ Pour Hirsch, " family life, even in its most intimate moments, is entrenched in a collective imaginary shaped by public, generational structures of fantasy and projection and by a shared archive of stories and images that inflect the broader transfer and availability of individual and familial remembrance ». HIRSCH Marianne, The Generation of Postmemory: Writing and Visual Culture After the Holocaust, New York, Columbia University Press, 2012, p. 35.

${ }^{27} \mathrm{Par}$ exemple, de 1993 à 2011 , le pourcentage des plus de 60 ans fréquentant le cinéma est passé de $27,7 \%$ à $57,3 \%$. Aujourd'hui, on estime que le pourcentage des spectateurs de plus de 50 ans $(33,6 \%)$ est plus important que celui des moins de 25 ans (31, $3 \%$ ). Pour ces chiffres et une mise en contexte de l'enquête du CNC, voir MANDelBaum Jacques, "Cinéma. Le grand âge d'or », Le Monde, 6 juillet 2013, p. 1 et p. 3. 
bot du spectateur sépia, on peut supposer que celui-ci est suffisamment âgé pour partager un cadre mémoriel commun avec les films.

Ce cadre mémoriel n'est pas seulement articulé autour de la mise en scène d'un passé familial. Entrent aussi en jeu les références auxquelles le film fait allusion et c'est ici que la dimension intertextuelle que nous avons introduite précédemment prend tout son sens. Ainsi, la vision du Petit Nicolas ne réactive pas seulement la nostalgie des années 1950, mais aussi, pour une partie du public, le souvenir des lectures enfantines des ouvrages de Goscinny et de Sempé. Les spectateurs des nouvelles versions de La Guerre des boutons se souviendront peut-être avec bonheur du film d'Yves Robert (1962) et plus généralement du cinéma populaire diffusé en salle à la fin des années 1950 et que l'on avait l'habitude d'aller voir en famille, lors de la traditionnelle sortie du samedi soir.

Si l'on continue de vouloir cerner le profil des spectateurs du cinéma sépia, peut-être doit-on ajouter que la motivation de ce public n'est pas seulement de retrouver un cadre mémoriel familier, mais aussi de le partager avec des générations plus jeunes. Un indice permettant d'aller dans ce sens est la présence dans les films eux-mêmes d'un dialogue intergénérationnel structurant la représentation autour d'une relation parents-enfants. Cette relation est visible dans Le Petit Nicolas, mais aussi dans Les Choristes où Gérard Jugnot fait figure de père de substitution. Des enquêtes plus affinées devraient être menées dans cette direction, mais on peut avancer que les films de la vague sépia sont avant tout destinés à un public familial et qu'ils sont le lieu d'une véritable transmission générationnelle. Ces productions peuvent réunir parents et enfants et constituent même le genre de film devant lequel on imagine très bien un grand-père emmener son petit-fils ou sa petite-fille. Et dès lors, on peut tout à fait concevoir que ces films vus en salle, mais peut-être aussi et surtout dans un espace domestique, lors du visionnement du DVD ou de la diffusion à la télévision $^{28}$, ont fonctionné comme une caisse de résonance qui a conduit la cellule familiale à évoquer son propre passé, à pointer les valeurs qui la fondent ou devraient la fonder.

Dans tous les cas, le travail d'appropriation mémorielle ne peut être dégagé de sa composante nostalgique. L'association entre mémoire centripète et nostalgie restauratrice n'est pas juste une association commode entre deux termes; elle permet d'expliquer au contraire

${ }^{28}$ Lors de sa première diffusion à la télévision française en 2006, Les Choristes a rassemblé environ 11,5 millions de spectateurs, dépassant ainsi les chiffres de fréquentation en salle. Pour plus de détails à ce sujet, voir le site lintern@ute.com, page disponible à l'adresse suivante : http://www.linternaute.com/ (consultée le 5 mai 2014). 
que l'une et l'autre se renforcent mutuellement. Si la nostalgie restauratrice ne peut franchir l'abîme du temps pour restaurer la patrie perdue, elle peut néanmoins compter sur la mémoire pour donner cette illusion. Plus le souvenir sera présenté comme intime et cohérent, plus la nostalgie restauratrice obéira à sa fonction première qui est de faire briller un paradis perdu, de le faire sentir presque à portée de main. Le souvenir d'une France idéalisée se trouve donc intériorisé par chaque spectateur au sein d'un cadre familial qui en assure l'unité et la cohésion.

\section{Le cinéma sépia en regard de la politique mémorielle de Nicolas Sarkozy}

Pour passer au niveau d'articulation suivant, la question que nous devons résoudre est celle de la fonction sociale des films dans la France des années 2000 , surtout en regard de la politique mémorielle menée sous la présidence de Nicolas Sarkozy à partir de 2007. Ces films sont-ils simplement le reflet d'une entreprise de mémoire qui s'accomplit au même moment selon des modalités similaires au niveau officiel de l'État? Ou, au contraire, y a-t-il discordance entre la sphère culturelle et la sphère politique dans la façon de gérer la mémoire du passé national?

Il est important de souligner que la plupart des films de la vague sépia possèdent, à un niveau implicite au moins, une fonction discriminante qui découle directement de la promotion d'une mémoire centripète véhiculée par des films jouant sur des références intertextuelles liées à un certain habitus culturel. Dans son essai Après le colonialisme, Arjun Appadurai a bien montré le caractère excluant de certains discours nostalgiques. Pour l'auteur, la patine (mais on pourrait parler dans les mêmes termes de la composante sépia) est "une ressource vraiment rare, car elle est le signe certain qu'un mode de vie a désormais disparu pour toujours. Pourtant, ce fait même est une garantie contre les nouveaux venus, car ils peuvent acquérir des objets patinés, mais jamais l'angoisse subtile de ceux qui peuvent légitimement se lamenter de la perte d'un mode de vie ${ }^{29}$. En ce sens, les films de la vague sépia s'inscrivent clairement dans une France marquée par la peur de l'Autre et le déploiement d'une politique sécuritaire de plus en plus importante coïncidant avec l'arrivée de Nicolas Sarkozy au ministère de l'Intérieur en 2005 , soit l'année suivant le triomphe des Choristes. 
Sur un plan strictement mémoriel, il serait tentant de faire du cinéma sépia la caisse de résonance des décisions prises en matière de commémorations ou de célébrations dans le champ politique. Tout autant que les films sépia, la politique menée par le gouvernement Sarkozy vise à promouvoir le souvenir d'une France idéale et à éluder les éléments douloureux et tragiques qui pourraient venir écorner cette image. Ainsi, lors de sa campagne présidentielle, Nicolas Sarkozy entendait se démarquer de son prédécesseur Jacques Chirac qui avait reconnu les torts de l'État français dans la déportation et les rafles menées à l'encontre de la population juive, notamment au Vel d'Hiv ${ }^{30}$. Plutôt qu'une mémoire tournée vers la reconnaissance des souffrances endurées et infligées, Nicolas Sarkozy souhaitait prôner un retour au roman national classique et mettre un terme à la " politique de repentance ${ }^{31}$ déployée par son prédécesseur. Pour l'historien Patrick Garcia, cette posture de non-repentance visait aussi «à rallier les secteurs de la population pour lesquels tout retour sur les drames du passé national est perçu comme une violence faite à la France et à eux-mêmes ${ }^{32}$.

Une fois élu président, Nicolas Sarkozy fut contraint de sacrifier nombre de ses ambitions en matière de politique mémorielle, ainsi qu'en atteste l'abandon du rapport Kaspi qui prévoyait dans la droite ligne de la campagne de ramener à trois dates le calendrier commémoratif ${ }^{33}$. Pareillement, de nombreuses initiatives mémorielles déployées par le nouveau gouvernement seront marquées par un échec cinglant. Ainsi, les principaux projets lancés par Nicolas Sarkozy ont-ils reçu une fin de non-recevoir des milieux autorisés, qu'il s'agisse des enseignants, des associations d'anciens combattants ou des membres de la société civile. Parmi les mesures envisagées par Sarkozy, on peut citer la volonté d'imposer aux enseignants que la dernière lettre du jeune Guy Môquet soit lue en début d'année à tous les lycéens de France (16

\footnotetext{
30 Dans le même temps qu'il reconnaissait les torts de l'État français dans la déportation de la population juive, Jacques Chirac faisait aussi valoir la solidarité des Justes de France, des personnes anonymes qui s'étaient dévouées pour sauver une grande partie de cette population. À ce sujet, voir GENSBURGER Sarah, Les Justes de France. Politiques publiques de la mémoire, op. cit.

${ }^{31}$ Voir, par exemple, le discours de Nicolas Sarkozy lors de son élection comme président de la République le 6 mai 2007, discours repris sur le site de Libération et disponible à l'adresse suivante : http://www.liberation.fr/ (consultée le 5 mai 2014).

${ }^{32}$ GARCIA Patrick, "Sarkozy a eu un rapport malheureux avec l'Histoire ", entretien donné dans Les Inrockuptibles, juillet 2012, disponible à l'adresse suivante : http://www.lesinrocks.com/ (consultée le 5 mai 2014).

${ }^{33}$ Réflexion sur la modernisation des commémorations publiques, le rapport Kaspi est téléchargeable à l'adresse suivante : http://ladocumentationfrancaise.fr. (rapport consulté le 14 août 2014).
} 
mai 2007$)^{34}$ ou l'idée d'instituer une Maison de l'histoire de France qui se serait implantée dans les locaux des Archives nationales (13 janvier 2009), sans oublier l'initiative vite abandonnée consistant à confier à chaque élève de CM2 la mémoire d'un enfant français victime de la Shoah (13 février 2008)35. Toutes ces initiatives seront frappées d'échec ou entraîneront de nombreuses polémiques, à l'image du débat sur l'identité nationale, lancé par le ministre de l'Intérieur Éric Besson en 2009. Au final, si le fondement de la politique mémorielle de Nicolas Sarkozy était de réenchanter le roman national, il faut bien reconnaître que cette politique fut loin de remporter le succès escompté.

\section{Les films sépia, un mécanisme compensatoire ?}

Face aux échecs répétés de la politique officielle, incapable de traduire en actions ses déclarations d'intention, on pourrait penser que les films de la vague sépia viennent occuper la place laissée vacante au niveau de l'État ${ }^{36}$. Ce qui ne peut se manifester en termes de cérémonies officielles trouve à s'exprimer dans des films comme La Nouvelle guerre des boutons, Le Petit Nicolas ou Les Choristes. Sans ironie aucune, on peut considérer qu'un film comme La Nouvelle guerre des boutons répond quasiment point par point aux directives mémorielles

${ }^{34}$ La polémique porta ici sur l'instrumentalisation de la mémoire entourant le sort de Guy Môquet. Le jeune homme fut célébré comme un résistant, alors que son emprisonnement et son exécution étaient liés à son engagement communiste et non à des actes de résistance en tant que tels. À ce sujet, comme pour l'ensemble de la politique mémorielle de Nicolas Sarkozy, le lecteur se rapportera à l'ouvrage de l'historien Nicolas Offenstadt, L'histoire bling-bling. Le retour du roman national. OfFENSTADT Nicolas, L'histoire blingbling. Le retour du roman national, Paris, Stock, 2009, 148 p. Au sujet de ces mêmes initiatives mémorielles, voir également GARCIA Patrick, "Nos présidents face à l'histoire », entretien donné dans Le Monde 2, mars

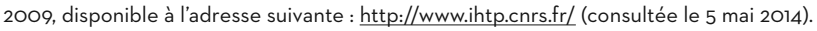

${ }^{35}$ Comme pour la célébration de Guy Môquet, cette initiative est révélatrice d'une approche de la mémoire qui privilégie avant tout l'impact émotionnel et une conception déhistoricisée de l'histoire. Cette proposition fut également vivement critiquée en raison de son effet éventuel sur la santé mentale des enfants. Rescapée d'Auschwitz et présidente d'honneur de la Fondation pour la mémoire de la Shoah, Simone Veil s'exprimera très clairement sur le sujet : «A la seconde où j'ai entendu ces mots, mon sang s'est glacé. Il est inimaginable, insoutenable, dramatique et, surtout, injuste d'infliger cela à des petits de 10 ans! On ne peut pas demander à un enfant de s'identifier à un enfant mort. Cette mémoire est beaucoup trop lourde à porter ». Déclaration reprise à l'adresse suivante : http://www.cndp.fr/ (consultée le 14 août 2014).

${ }^{36}$ Même si nous ne retenons pas l'hypothèse d'une complémentarité instrumentale entre l'État et la vague sépia, il faut souligner l'existence d'un court métrage qui rapproche de manière concrète l'un des acteurs phares des films sépia et une initiative mémorielle issue de la présidence de Nicolas Sarkozy. En 2007, la chaîne parlementaire (LCP) et France Télévision produisent La Lettre, un court métrage signé François Hanss mettant en scène les derniers instants de Guy Môquet. Le rôle du jeune communiste est tenu par Jean-Baptiste Maunier qui était déjà l'un des interprètes principaux des Choristes de Christophe Barratier. Durant la deuxième quinzaine d'octobre 2007, La Lettre sera diffusé sur LCP-Assemblée Nationale, les chaînes de télévision nationale (France 2, France 3) et TV5. Une adaptation radiophonique sera également diffusée sur Radio France et le film sera projeté en permanence au Mémorial de Caen. Pour voir ce film et disponible à l'adresse suivante : http://www.zerodeconduite.net/ (consultée le 14 août 2014). 
énoncées par Nicolas Sarkozy. Si on ne lit pas la lettre de Guy Môquet, on célèbre tout de même bien le courage d'apprentis résistants et surtout, les jeunes héros du film réalisent ce qu'auraient dû accomplir les élèves de $\mathrm{CM}$ 2, à savoir adopter la mémoire d'un(e) enfant français(e) victime de la Shoah. Plus généralement, les autres films participent à dresser l'inventaire d'une Maison de l'histoire de France en cumulant les objets et les signes représentatifs d'une certaine idée de l'identité française.

Pour autant, il ne s'agit pas d'avancer ici que les films de Christophe Barratier ou de Laurent Tirard sont au service du pouvoir, chargés en sous-main d'accomplir ce que la politique officielle ne peut mener à bien. À mon sens, il serait inadéquat de penser la vague sépia comme un pur mécanisme de compensation, même s'il paraît probable que beaucoup de spectateurs, dont certains avaient sans doute voté pour Nicolas Sarkozy en 2007, ont trouvé dans les films le parfum envoûtant d'une France passée, le souvenir nostalgique d'un idéal identitaire que l'État était incapable de traduire en commémorations publiques.

Plutôt que de réfléchir en termes de compensation, il est plus intéressant d'essayer de comprendre comment le souvenir d'un même passé national peut se trouver critiqué ou plébiscité par des mêmes personnes selon qu'elles se trouvent dans le champ de l'action publique ou dans celui des représentations médiatiques. Pareille position revient à éviter de considérer le public du cinéma sépia comme une majorité silencieuse qui aurait trouvé refuge dans les films parce que sa voix ne pouvait être suffisamment entendue sur la place publique pour plébisciter la politique mémorielle de Nicolas Sarkozy. Selon moi, une hypothèse plus forte est de soutenir que ce n'est pas tellement le passé national qui fait ou non l'objet d'un plébiscite, mais plutôt les cadres mémoriels chargés de l'exprimer. Dans cette perspective, on peut tout à fait avancer que de nombreux citoyens opposés à la politique mémorielle de Nicolas Sarkozy constituaient, en même temps, une large part du public ayant fait le succès des films de la vague sépia.

Cette ambivalence des attitudes mémorielles trouve un répondant théorique dans ce que l'anthropologue Michael Herzfeld appelle des situations de disémie, à savoir des situations révélant une certaine tension " entre une présentation officielle de soi et ce qui surgit dans

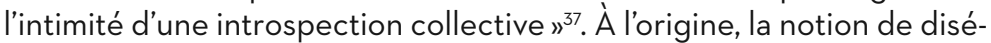
mie est utilisée en sociolinguistique pour distinguer les formes officielles du langage de ses usages vernaculaires, et surtout pour mettre en avant la capacité qu'ont certains locuteurs de passer d'un registre

${ }^{37}$ HerzFeld Michael, Cultural Intimacy. Social Poetics in the Nation-State, New York, Routledge, 2005, p. 14 [traduction de l'auteur]. 
à l'autre. Chez Herzfeld, la notion déborde le domaine linguistique et vise à éclairer la façon dont les registres officiel et populaire peuvent faire l'objet d'usages subversifs et ironiques dans la construction de l'identité culturelle des individus.

Pour ma part, il me semble que la notion de disémie permet d'attirer l'attention sur la tension ou la rivalité pouvant surgir entre des cadres mémoriels différents. De la même manière qu'il existe une tension entre les registres élevé et populaire, on peut supposer qu'il existe une tension entre le cadre mémoriel officiel fourni par l'État-nation et celui, plus intime, établi par la mémoire familiale, encore que des jeux de transfert et d'appropriation puissent s'effectuer d'un cadre à l'autre. Pour Herzfeld, il arrive fréquemment que l'État utilise des métaphores relevant de l'univers domestique pour asseoir ses discours officiels, ce qui se vérifie en certains points de la politique mémorielle de Nicolas Sarkozy. Ainsi, les grandes initiatives évoquées précédemment privilégient les références au cadre familial, ne serait-ce que par les choix sémantiques qui se retrouvent dans les projets d'adopter la mémoire d'un enfant de la Shoah ou de construire une Maison de l'histoire de France.

Mais le point essentiel réside à mon sens dans le fait que la tension entre cadres mémoriels ne présuppose pas des contenus nécessairement différents. Au contraire, un même contenu mémoriel peut voyager d'un cadre à l'autre, se trouver configuré différemment en fonction du cadre dans lequel il s'exprime et susciter alors un plébiscite plus ou moins appuyé à l'intérieur de ce contexte donné. Ce détour théorique permet de comprendre comment une part du public français des années 2000 affiche un intérêt marqué pour son passé national, sans partager pour autant les initiatives mémorielles délivrées par l'État. Les films de la vague sépia ont ainsi permis à des spectateurs de cultiver, à l'abri de la salle obscure, une nostalgie restauratrice qu'ils étaient loin d'approuver en public, certains pouvant même être nettement opposés aux initiatives proposées par le gouvernement Sarkozy. Et l'on peut avancer que cette nostalgie restauratrice était d'autant mieux approuvée qu'elle ne se proposait pas sur le mode de l'action politique, mais sous la forme du divertissement et du spectacle familial.

\section{D'un cadre mémoriel à l'autre}

Pour conforter cette hypothèse, retournons aux films de la vague sépia et observons tout d'abord que ceux-ci se caractérisent par une défiance face aux institutions et aux grandes structures de pouvoir. Cela apparaît clairement dans Les Choristes où Gérard Jugnot transforme de l'intérieur et, pour un temps seulement, un pensionnat qui a 
des airs de véritable prison. Faubourg 36 valorise les luttes des classes populaires avec des personnages qui se battent contre un promoteur de spectacles incarnant à lui seul les méfaits de l'argent et du capitalisme. Dans Le Petit Nicolas, c'est un spectacle imposé par le ministère de l'Éducation qui suscite le découragement de l'institutrice. Bref, à bien y regarder, tous les films expriment une méfiance latente à l'égard des structures officielles et des positions hiérarchiques élevées, ce qui justifie d'autant plus la priorité donnée à la sphère familiale et privée.

Ce trait participe déjà à autonomiser le cadre mémoriel des films, puisque ceux-ci ne célèbrent en rien les institutions dont l'État est normalement le garant. Mais cette méfiance à l'égard du pouvoir renvoie aussi à la position des cinéastes eux-mêmes dans le champ cinématographique. Sans entrer dans une analyse détaillée de ce champ socio-professionnel, on peut constater que des réalisateurs comme Christophe Barratier, Laurent Tirard ou Yann Samuell ne sont pas tenus en haute estime par les critiques spécialisés ou par certaines institutions officielles chargées de célébrer le cinéma français. II faut se rappeler que Les Choristes a constitué un succès-surprise en 2004 et que personne ne s'attendait à un tel résultat, à commencer par Christophe Barratier lui-même dont c'était le premier long-métrage. À la cérémonie des Césars, le film sera nominé, mais ne repartira qu'avec deux Césars de circonstance. On assiste à un cas de figure identique pour Le Petit Nicolas qui, bien que plus grand succès français au box-office français en 2009, ne recevra pas la moindre récompense lors de la cérémonie récompensant les meilleures productions françaises de l'année ${ }^{38}$. Autrement dit, il y a une homologie entre la position des réalisateurs dans le champ cinématographique et la méfiance que les films expriment à l'égard des institutions. Cette homologie n'est pas sans produire d'effets, car elle permet d'une part, de renforcer auprès des spectateurs l'image réductrice d'un cinéma populaire caractérisé par un important succès public, mais méprisé par les élites, et d'autre part, d'affranchir le cadre mémoriel véhiculé par les productions sépia de toute compromission avec des instances de légitimation, qu'elles soient cinématographiques ou politiques.

Une autre différence par rapport à la mémoire officielle est que les films de la vague sépia ne se font pas l'écho d'une grande intrigue nationale qui serait reprise de film en film avec certaines variantes. Dans son ouvrage sur la nostalgie, Svetlana Boym a bien insisté sur la

${ }^{38}$ Le problème de la non-reconnaissance des films populaires sera explicitement formulé par Dany Boon, lorsque son film Bienvenue chez les Ch'tis (2009) ne sera pas sélectionné pour la cérémonie des Césars. En cette occasion, le réalisateur menaça de boycotter la cérémonie et proposa également la création d'un César de la Meilleure comédie. 
différence entre mémoire collective et mémoire nationale. Ces deux mémoires sont distinctes, explique-t-elle, même si elles peuvent partager un ensemble d'images et citations. Alors que la mémoire collective ne présuppose pas une narration parfaitement structurée, la mémoire nationale est bâtie sur le modèle de la prescription et tend «à produire une seule intrigue téléologique à partir des souvenirs partagés de la vie quotidienne ${ }^{39}$. Clairement, les films sépia ne fonctionnent pas sur ce modèle. Au contraire, ceux-ci sont incapables de produire un récit de grande ampleur puisque, comme nous l'avons vu, l'intrigue déborde rarement le cadre du foyer domestique ou de la sphère privée. Par ailleurs, plus qu'à un agencement narratif performant ou à une rigoureuse progression dramatique, les films renvoient davantage à une succession de clichés épars, une accumulation de sketchs qui priment parfois sur l'avancée de l'histoire elle-même.

En conclusion, et c'est en ce point que se noue le travail d'articulation entrepris sous l'égide de Stuart Hall, les films sépia nous renseignent autant sur ce qui a été perdu que sur ce qui pourrait être retrouvé. Ce qui a été perdu, semblent dire les films, c'est la confiance dans les pouvoirs établis, dans une grande histoire qui ferait sens pour tous; ce qui pourrait être retrouvé, semblent-ils dire aussi, c'est le sens de la famille et des valeurs traditionnelles qui n'ont pas nécessairement besoin du cadre étatique pour se manifester. Par conséquent, on comprend mieux l'impact des films sépia dans le paysage français des années 2000. Dans le contexte d'une absence de politique mémorielle fédératrice menée au niveau de l'État, ces films expriment une désarticulation du passé national qui ne trouve plus qu'à s'exprimer sous forme de vignettes quotidiennes, le cadre familial et domestique remplaçant le cadre de l'État-nation dans la ré-articulation et la réappropriation des souvenirs ayant trait au passé collectif. 